EISSN: 2706 -7947 ISSN: 2077- 4613

DOI: 10.36632/mejas/2021.11.4.69

Journal homepage: www.curresweb.com

Pages: 887-895

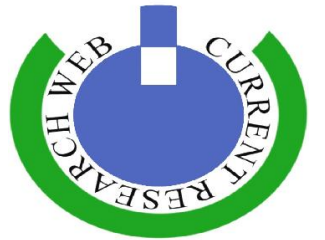

\title{
Effect of mineral and organic fertilizers on yield, yield components and chemical composition of some wheat cultivars
}

\section{Hassanein M. S., Nabila M. Zaki and Amal G. Ahmed}

Field Crops Research Department, National Research Centre, 33 El-Bohouth St., Dokki, Giza, Egypt. Postal Code: 12622.

Received: 12 Oct. $2021 \quad$ Accepted: 05 Nov. $2021 \quad$ Published: 10 Nov. 2021

\begin{abstract}
Two field experiments were carried out during 2017/2018 and 2018/2019 seasons at Wadi El- Rayan Fayoum Governorate, Egypt to study the effect of nitrogen fertilizer (ammonia gas 82\%) and organic fertilizer on yield and its components of two wheat cultivars. The main results could be summarized as follows: Misr-1 cultivar exceed Sids-1 cultivar in grain yield and its components significantly except grain yield (ton/fed.) and harvest index\%. Addition of $75 \mathrm{~kg} \mathrm{~N} /$ feddan (ammonia gas $82 \%$ ) resulted in a significant increment in yield and its components except harvest index\% comparison with other treatments (control and $50 \mathrm{~kg} \mathrm{~N} /$ feddan). The application of organic fertilizer at the rate of $15 \mathrm{ton} /$ feddan significantly increased yield and its components, except harvest index\%. The interaction between wheat cultivars and nitrogen fertilizer was significant yield and its components except grain index $(\mathrm{g})$, straw yield ton/feddan, protein percentage and carbohydrate percentage, while the interaction between wheat cultivars and organic fertilizer was significant in all characters under study except number of tillers $/ \mathrm{m}^{2}$, grain index and straw yield (ton/ feddan). The interaction between nitrogen fertilizer and organic fertilizer was significant in all characters under study except number of spikes $/ \mathrm{m}^{2}$ and straw yield (ton/ feddan). It is clear from results that the interaction between wheat cultivars $\mathrm{x}$ nitrogen fertilizer $\mathrm{x}$ organic fertilizer was significant in all characters under study except number of spikes $/ \mathrm{m}^{2}$, straw yield (ton/ feddan) and protein percentage $\%$. The most effective treatment were obtain by using Misr-1 cultivar $+75 \mathrm{~kg} \mathrm{~N} /$ fed., +15 ton / fed., organic fertilizer.
\end{abstract}

Keywords: Wheat, cultivars, nitrogen, ammonia gas, organic, fertilizer, yield and yield components.

\section{Introduction}

Wheat (Triticum aestivum L.) is considered to be one of the most widely grown crops in high nutritive value in the world. The grains of wheat contain large amounts of proteins, carbohydrates in addition to some mineral and vitamins. Wheat has a special importance because the local production is not sufficient to meet the annual demands. Increasing the local production is a target to cover the local consumption. This could be achieved by introducing more productive varieties, improving the culture practices such as sowing the wheat in the newly reclaimed area or application of some promoters during different growth stages. Recently, a great attention of several investigation has been directed to increase the productivity of wheat to minimize the gap between the Egyptian production and consumption by increasing the cultivated area and wheat yield per unit area.

The total biomass is a result of the integration of metabolic reaction in the plants. Consequently any factor influencing the metabolic activity of the plant at any period of its growth can affect the yield. Metabolic processes in wheat plants are greatly governed by both internal i.e. genetic makeup of the plant and external conditions which namely climatic and edaphically environmental factors. Thus, increasing wheat production per unit area can be achieved by breeding and cultivating the promising

Corresponding Author: Hassanein M.S., Field Crops Research Department, National Research Centre, 33 ElBohouth St., Dokki, Giza, Egypt. Postal Code: 12622.

E-mail: mosaad.soliman@yahoo.com 
wheat cultivars and applying the optimum cultural practices such as suitable fertilizer. El-Esh (2007) reported that there significant differences among the three cultivars in all characters under study. There were a significant differences among cultivars (Zaki et al, 2012, Zaki et al, 2016, Abd El-All et al, 2017, Wali et al, 2018, Hassanein et al, 2019 and Zaki et al, 2019 ). Nutrition is essential for plant life and yield therefore; mineral fertilization is a common agronomic practice that leads to improve productivity. But with the steadily increasing prices of chemical fertilizers especially nitrogen fertilizers and the pollution problems of soil and water. The organic fertilizer is becoming more important, Hosam El-Din (2007) and Zaki et al (2012) the application of farmyard manure at different rates significantly increased yield and its components were positively affected by adding the organic manure at the different rates. Zeidan et al. (2009) reported that nitrogen fertilization with or without addition of organic fertilizer significantly affected characters under study. Organic fertilization reduce pollution and sustain soil fertility through their effect on the physical, chemical and biological properties of soil, but when it's use alone is not sufficient to meet the requirement of nutrients.

The aim of this investigation was designed to study the effect of mineral and organic fertilizer on yield, yield components and chemical composition in two wheat cultivars.

\section{Material and Methods}

Two field experiments were conducted at new land at Wadi El-Rayan, El- Fayoum Governorate, Egypt during 2017/2018 and 2018/2019 seasons. The experiments were carried out to study the combined effect of mineral nitrogen fertilizer i.e. (ammonia gas $82 \%$ ) and organic fertilizer on yield, yield components and chemical composition of two wheat cultivars (Triticum aestivum L.). The physical and chemical characters of soil (30 cm depth) in the experimental site were as follows; sand $72.59 \%$, silt $23.47 \%$, clay $3.45 \%$, PH 8.00 , organic matter $0.84 \%$, soluble N 84.00 ppm, soluble P $13.50 \mathrm{ppm}$, and soluble K $132.00 \mathrm{ppm}$., according to as described by Chapman and Pratt (1961).

The experimental design was split-split plot design with four replications. Wheat cultivars were allocated randomly in the main plots (Sids-1 and Misr-1), nitrogen fertilizer were randomly allocated in sub- plots (Control, $50 \mathrm{~kg} \mathrm{~N} /$ fed., and $75 \mathrm{~kg} \mathrm{~N} / \mathrm{fed}$.). Organic fertilizer were randomly allocated in sub-sub plot ( 5 ton/ fed., 10 ton/ fed., and 15 ton/ fed., chicken manure). The size of each plot was $10.5 \mathrm{~m}^{2}$ (1/400 feddan) $3.5 \mathrm{~m}$ long and $3 \mathrm{~m}$ wide. Each experiment included 18 treatments which were the combination among two cultivars, three nitrogen level and three organic fertilizer level. Nitrogen fertilizer added at the first irrigation and 25 days after the first irrigation in form of ammonia gas. Super phosphate fertilizer $\left(15.5 \% \mathrm{P}_{2} \mathrm{O}_{5}\right)$ was applied before sowing at the rate of $150 \mathrm{~kg} / \mathrm{fed}$., also organic fertilizer form chicken manure was added and mixed with the soil two weeks before sowing raked it lightly at a depth of $10-15 \mathrm{~cm}$. Potassium fertilizer was applied before sowing at a rate of $50 \mathrm{~kg} / \mathrm{fed}$., in the form of potassium sulphate $\left(48 \% \mathrm{~K}_{2} \mathrm{O}\right)$. Sowing date were November $15^{\text {th }}$ and November $20^{\text {th }}$ in both seasons, respectively, while, seeding rate was $70 \mathrm{~kg} /$ fed. The normal agronomic practices of wheat were followed until harvest as recommended by Wheat Research Dep., Agric. Research Centre.

At harvest, 10 plants at random were taken from each plot to determine plant height $(\mathrm{cm})$ and grain index $(\mathrm{g})$,also, one square meter was taken from each plot to determine number of tillers $/ \mathrm{m}^{2}$, number of spikes $/ \mathrm{m}^{2}$, weight of spikes $\left(\mathrm{g} / \mathrm{m}^{2}\right)$ and grain yield $\left(\mathrm{g} / \mathrm{m}^{2}\right)$. Straw yield (ton/fed), grain yield (ton/fed), biological yield (ton/fed) and harvest index\% was estimated from each plot. Grain protein content was estimated as $\mathrm{N} \% \times 5.75$ on dry weight basis where nitrogen $\%$ was determined by the micro - kjeldahl methods according to A.O.A.C., (1988). Carbohydrate \% was determined according to Dubois et al. (1956).

Data obtained were exposed to the proper method of statistical analysis of variance differentiate among means of different treatments as described by Gomez and Gomez (1984). The treatments means were compared using the least significant differences (L.S.D.) test at $5 \%$ level of probability. Combined analysis was made from the two growing seasons hence the results of two seasons followed similar trend. 


\section{Results and Discussion}

\subsection{Yield, its components and chemical composition: I. Effect of wheat cultivars:}

Data in Table (1) revealed that wheat cultivars Sids-1 and Misr-1 differ significantly in yield, its components and chemical composition i.e. plant height $(\mathrm{cm})$, number of tillers $/ \mathrm{m}^{2}$, number of spikes/ $\mathrm{m}^{2}$, weight of spikes $\left(\mathrm{g} / \mathrm{m}^{2}\right)$, grain yield $\left(\mathrm{g} / \mathrm{m}^{2}\right)$, grain index $(\mathrm{g})$, straw yield (ton/fed.), biological yield (ton/fed.), protein $\%$ and carbohydrate $\%$ except grain yield (ton/fed.) and harvest index in both seasons. Misr-1 cultivar significantly surpassed Sids-1 cultivar at all above characters except grain yield (ton/fed.) and harvest index in both seasons.

It could be concluded that varietal differences between wheat cultivars may be due to genetically differences between cultivars, as well as, rang of cultivars response. It noteworthy to mention that differences in yield potential of wheat depend undoubtedly on the part of photosynthesis partitioned into grain yield. However, this depends on source size and translocation, as, Abd El-Gawad et al (1987) indicated that wheat cultivars differed in partitioning and migration of the total available photosynthetic to economic yields. Also, wheat cultivars differed in carbon equivalent for vegetative components and grain and in production value of vegetative matter, grains / plant and / fed, and coefficient energy of crop and harvest index.

These results were in harmony with the results obtained by El-Esh (2007), Suzan (2007), Zaki et al (2012), Ahmed et al (2013), Hassanein et al (2013), Zaki et al (2015), Abd El-All et al (2017), Wali et al (2018), Zaki et al (2018), Hassanein et al (2019) and Zaki et al (2019) .

\section{Effect of nitrogen fertilizer:}

Data presented in Table (1) showed that increasing nitrogen fertilizer (ammonia gas ) significantly affected all wheat characters i.e.( (plant height $(\mathrm{cm})$, number of tillers $/ \mathrm{m}^{2}$, number of spikes/ $\mathrm{m}^{2}$, weight of spikes $\left(\mathrm{g} / \mathrm{m}^{2}\right)$, grain yield $\left(\mathrm{g} / \mathrm{m}^{2}\right)$, grain index $(\mathrm{g})$, straw yield (ton/fed.), grain yield (ton/fed.), biological yield (ton/fed.), protein $\%$ and carbohydrate $\%$ ) on both seasons except harvest index. Such increase in plant height may be occurred due to the stimulation of cell division and internodes elongation resulted from nitrogen fertilizer (ammonia gas). These results are in harmony with those reported by Shoman et al. (2006), Zaki et al. (2012) Hassanein et al. (2013), Abd El-All et al. (2017), Wali et al. (2018) and Zaki et al. (2019).

Data clear that increasing nitrogen fertilizer significantly increased grain, straw and biological yield (ton/fed) in both seasons. This increase in grain yield (ton/fed.) could be attributed to the significant increase in number of spikes $/ \mathrm{m}^{2}$, weight of spikes $\left(\mathrm{g} / \mathrm{m}^{2}\right)$, grain yield $\left(\mathrm{g} / \mathrm{m}^{2}\right)$ and grain index (g). These finding are in agreement with those obtained by Zeidan et al. (2009) Hassanein et al. (2013) and Hassanein et al. (2018).

In addition, the increase in grain yield and other studied traits could be due to the increase in dry weight of vegetative organs which might consider as a criterion for the photosynthetic efficiency of the plant (Sabah, 2006 and Zaki et al, 2012).

In general, application of nitrogen fertilizer (ammonia gas) at rate of $75 \mathrm{~kg} \mathrm{~N} /$ fed., gave the highest yield and its components compared to $50 \mathrm{~kg} \mathrm{~N} / \mathrm{fed}$., and to control.

\section{Effect of organic fertilizer:}

Data in Table (1) indicated that adding organic fertilizer at rate of 15 ton / fed., gave the highest value of all characters i.e.( (plant height $(\mathrm{cm})$, number of tillers $/ \mathrm{m}^{2}$, number of spikes/ $\mathrm{m}^{2}$, weight of spikes $\left(\mathrm{g} / \mathrm{m}^{2}\right)$, grain yield $\left(\mathrm{g} / \mathrm{m}^{2}\right)$, grain index $(\mathrm{g})$, straw yield (ton/fed.), grain yield (ton/fed.), biological yield (ton/fed.), harvest index, protein $\%$ and carbohydrate $\%$ ) under study followed by 10 ton / fed., and 5 ton / fed.,. The differences between organic fertilizer levels were significant for all characters under study in both seasons. This superiority of 15 ton / fed., organic fertilizer may be due to the stimulate effect of it through flowering and grain production (Sadur et al., 2008 and Kabesh et al., 2009).

\section{Effect of interaction:}

The effect of interaction between wheat cultivars and nitrogen fertilizer (ammonia gas) on plant height $(\mathrm{cm})$, number of tillers $/ \mathrm{m}^{2}$, number of spikes $/ \mathrm{m}^{2}$, weight of spikes $\left(\mathrm{g} / \mathrm{m}^{2}\right)$, grain yield $\left(\mathrm{g} / \mathrm{m}^{2}\right)$, 
Table 1: Effect of cultivars, nitrogen fertilizer and organic fertilizer on yield, its components and chemical composition of wheat (Average of 2017/2018 and 2018/2019 seasons).

\begin{tabular}{|c|c|c|c|c|c|c|c|c|c|c|c|c|}
\hline $\begin{array}{l}\text { Characters } \\
\text { Treatments } \\
\end{array}$ & $\begin{array}{c}\text { Plant } \\
\text { height } \\
\text { (cm) }\end{array}$ & $\begin{array}{c}\text { No. of } \\
\text { tillers } \\
\left(\mathbf{m}^{2}\right) \\
\end{array}$ & $\begin{array}{c}\text { No. of } \\
\text { spikes } \\
\left(\mathbf{m}^{2}\right)\end{array}$ & $\begin{array}{c}\text { Weight } \\
\text { of spikes } \\
\left(\mathrm{g} / \mathrm{m}^{2}\right) \\
\end{array}$ & $\begin{array}{c}\text { Gain } \\
\text { yield } \\
\left(\mathrm{g} / \mathrm{m}^{2}\right) \\
\end{array}$ & $\begin{array}{c}\text { Grain } \\
\text { index } \\
(\mathrm{g}) \\
\end{array}$ & $\begin{array}{c}\text { Straw } \\
\text { yield } \\
\text { (ton/fed) }\end{array}$ & $\begin{array}{c}\text { Grain } \\
\text { yield } \\
\text { (ton/fed) }\end{array}$ & $\begin{array}{c}\text { Biological } \\
\text { yield } \\
\text { (ton/fed) }\end{array}$ & $\begin{array}{c}\text { Harvest } \\
\text { Index } \\
\% \\
\end{array}$ & $\begin{array}{c}\text { Protein } \\
\%\end{array}$ & $\begin{array}{c}\text { Carbohydrate } \\
\%\end{array}$ \\
\hline \multicolumn{13}{|c|}{ Cultivars } \\
\hline Sids 1 & 120.36 & 540.70 & 516.91 & 778.22 & 574.67 & 44.17 & 8.731 & 5.208 & 13.939 & 37.36 & 11.50 & 82.40 \\
\hline Misr 1 & 126.00 & 544.13 & 526.59 & 797.49 & 582.04 & 46.17 & 8.900 & 5.277 & 14.177 & 37.31 & 11.55 & 85.60 \\
\hline L.S.D. at 5\% & 0.38 & 2.40 & 4.84 & 0.36 & 0.41 & 0.84 & 0.089 & n.s & 0.210 & n.s & 0.04 & 0.64 \\
\hline \multicolumn{13}{|c|}{ Nitrogen fertilizer(Ammonia Gas) } \\
\hline Control & 120.12 & 529.01 & 507.31 & 773.12 & 566.92 & 43.54 & 8.662 & 5.137 & 13.799 & 37.19 & 11.46 & 81.03 \\
\hline $50 \mathrm{~kg} \mathrm{~N} /$ fed. & 123.39 & 542.13 & 520.10 & 790.63 & 580.63 & 45.35 & 8.835 & 5.285 & 14.120 & 37.55 & 11.52 & 84.85 \\
\hline $75 \mathrm{~kg} \mathrm{~N} /$ fed. & 126.03 & 556.12 & 537.83 & 799.81 & 587.52 & 46.61 & 8.949 & 5.306 & 14.255 & 37.26 & 11.59 & 86.12 \\
\hline L.S.D. at 5\% & 0.10 & 2.21 & 2.90 & 0.53 & 0.52 & 0.28 & 0.068 & 0.049 & 0.070 & n.s & 0.01 & 0.44 \\
\hline \multicolumn{13}{|c|}{ Organic fertilizer } \\
\hline 5 ton/ fed. & 119.94 & 531.33 & 512.26 & 728.59 & 564.04 & 43.51 & 8.679 & 5.074 & 13.653 & 36.92 & 11.46 & 80.94 \\
\hline 10 ton/ fed. & 123.23 & 543.67 & 520.77 & 790.06 & 580.21 & 45.20 & 8.807 & 5.315 & 14.122 & 37.73 & 11.52 & 83.82 \\
\hline 15 ton/ fed. & 126.36 & 552.25 & 532.21 & 800.47 & 590.82 & 46.80 & 8.960 & 5.339 & 14.299 & 37.35 & 11.60 & 87.24 \\
\hline L.S.D. at 5\% & 0.14 & 1.87 & 2.52 & 0.61 & 0.50 & 0.25 & 0.053 & 0.044 & 0.082 & 0.28 & 0.02 & 0.30 \\
\hline
\end{tabular}

Values followed by the same letter (s) within column are not significantly different $(\mathrm{P}<0.05)$ 
Table 2: Effect of interaction between cultivars x nitrogen fertilizer on yield, its components and chemical composition of wheat (Average of 2017/2018 and 2018/2019 seasons).

\begin{tabular}{|c|c|c|c|c|c|c|c|c|c|c|c|c|c|}
\hline Treatr & Characters & $\begin{array}{c}\text { Plant } \\
\text { height } \\
(\mathrm{cm})\end{array}$ & $\begin{array}{c}\text { No. of } \\
\text { tillers } \\
\left(\mathbf{m}^{2}\right)\end{array}$ & $\begin{array}{c}\text { No. of } \\
\text { spikes } \\
\left(\mathbf{m}^{2}\right)\end{array}$ & $\begin{array}{c}\text { Weight } \\
\text { of } \\
\text { spikes } \\
\left(\mathrm{g} / \mathrm{m}^{2}\right) \\
\end{array}$ & $\begin{array}{c}\text { Gain } \\
\text { yield } \\
\left(\mathbf{g} / \mathbf{m}^{2}\right)\end{array}$ & $\begin{array}{c}\text { Grain } \\
\text { index } \\
(\mathrm{g})\end{array}$ & $\begin{array}{c}\text { Straw } \\
\text { yield } \\
\text { (ton/fed) }\end{array}$ & $\begin{array}{c}\text { Grain } \\
\text { yield } \\
\text { (ton/fed) }\end{array}$ & $\begin{array}{c}\text { Biological } \\
\text { yield } \\
\text { (ton/fed) }\end{array}$ & $\begin{array}{c}\text { Harvest } \\
\text { index } \\
\%\end{array}$ & $\begin{array}{c}\text { Protein } \\
\%\end{array}$ & $\begin{array}{c}\text { Carbohydrate } \\
\%\end{array}$ \\
\hline \multicolumn{14}{|c|}{ Cultivars x Nitrogen fertilizer (Ammonia Gas) } \\
\hline \multirow{3}{*}{$\begin{array}{l}\text { Sids } \\
1\end{array}$} & Control & 116.89 & 524.86 & 497.29 & 763.22 & 560.16 & 42,44 & 8.623 & 5.074 & 13.697 & 36.80 & 11.44 & 79.23 \\
\hline & 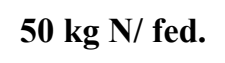 & 120.62 & 541.61 & 516.99 & 782.66 & 575.89 & 44.49 & 8.740 & 5.300 & 14.040 & 37.93 & 11.49 & 83.34 \\
\hline & $75 \mathrm{~kg} \mathrm{~N} /$ fed. & 123.57 & 555.64 & 536.44 & 788.78 & 587.98 & 45.57 & 8.830 & 5.250 & 14.080 & 37.33 & 11.56 & 84.61 \\
\hline \multirow{3}{*}{$\begin{array}{l}\text { Misr } \\
1\end{array}$} & Control & 123.34 & 533.17 & 517.32 & 783.02 & 573.68 & 44.64 & 8.701 & 5.200 & 13.901 & 37.59 & 11.48 & 82.83 \\
\hline & 50 kg N/ fed. & 126.16 & 542.64 & 523.21 & 798.60 & 585.38 & 46.21 & 8.930 & 5.270 & 14.200 & 37.17 & 11.56 & 86.36 \\
\hline & $75 \mathrm{~kg} \mathrm{~N} /$ fed. & 128.49 & 556.59 & 539.22 & 810.84 & 587.06 & 47.66 & 9.068 & 5.362 & 14.430 & 37.18 & 11.62 & 87.62 \\
\hline \multicolumn{2}{|c|}{ L.S.D. at 5\% } & 0.14 & 3.12 & 4.11 & 0.76 & 0.73 & n.s & n.s & 0.069 & 0.099 & 0.54 & n.s & n.s \\
\hline
\end{tabular}

Table 3: Effect of interaction between cultivars x organic fertilizer on yield and its components of wheat (Average of 2017/2018 and 2018/2019 seasons).

\begin{tabular}{|c|c|c|c|c|c|c|c|c|c|c|c|c|c|}
\hline Treatr & $\begin{array}{l}\text { Characters } \\
\text { ents }\end{array}$ & $\begin{array}{c}\text { Plant } \\
\text { height } \\
\text { (cm) }\end{array}$ & $\begin{array}{l}\text { No. of } \\
\text { tillers } \\
\left(\mathbf{m}^{2}\right)\end{array}$ & $\begin{array}{l}\text { No. of } \\
\text { spikes } \\
\left(\mathbf{m}^{2}\right)\end{array}$ & $\begin{array}{c}\text { Weight } \\
\text { of } \\
\text { spikes } \\
\left(\mathrm{g} / \mathrm{m}^{2}\right)\end{array}$ & $\begin{array}{c}\text { Gain } \\
\text { yield } \\
\left(\mathbf{g} / \mathbf{m}^{2}\right)\end{array}$ & $\begin{array}{c}\text { Grain } \\
\text { index } \\
(\mathrm{g})\end{array}$ & $\begin{array}{c}\text { Straw } \\
\text { yield } \\
\text { (ton/fed) }\end{array}$ & $\begin{array}{c}\text { Grain } \\
\text { yield } \\
\text { (ton/fed) }\end{array}$ & $\begin{array}{c}\text { Biological } \\
\text { yield } \\
\text { (ton/fed) }\end{array}$ & $\begin{array}{c}\text { Harvest } \\
\text { index } \\
\%\end{array}$ & $\begin{array}{c}\text { Protein } \\
\%\end{array}$ & $\begin{array}{c}\text { Carbohydrate } \\
\%\end{array}$ \\
\hline \multicolumn{14}{|c|}{ Cultivars $x$ Organic fertilizer } \\
\hline \multirow{3}{*}{$\begin{array}{c}\text { Sids } \\
1\end{array}$} & 5 ton $/$ fed. & 116.84 & 529.26 & 510.46 & 763.37 & 562.27 & 42.48 & 8.600 & 4.951 & 13.551 & 36.52 & 11.44 & 78.46 \\
\hline & 10 ton/ fed. & 120.12 & 541.28 & 515.68 & 778.78 & 574.27 & 44.14 & 8.736 & 5.334 & 14.070 & 37.97 & 11.48 & 82.38 \\
\hline & 15 ton/ fed. & 124.11 & 551.58 & 524.59 & 792.51 & 587.49 & 45.88 & 8.858 & 5.339 & 14.197 & 37.58 & 11.57 & 86.36 \\
\hline \multirow{3}{*}{$\begin{array}{c}\text { Misr } \\
1\end{array}$} & 5 ton/ fed. & 123.03 & 533.41 & 514.07 & 782.70 & 565.81 & 44.53 & 8.759 & 5.197 & 13.956 & 37.32 & 11.48 & 83.42 \\
\hline & 10 ton/ fed. & 126.34 & 546.07 & 525.87 & 801.33 & 586.14 & 46.26 & 8.878 & 5.296 & 14.174 & 37.49 & 11.56 & 85.26 \\
\hline & 15 ton/ fed. & 128.61 & 552.92 & 539.82 & 808.43 & 594.16 & 47.72 & 9.062 & 5.340 & 14.402 & 37.12 & 11.63 & 88.13 \\
\hline \multicolumn{2}{|c|}{ L.S.D. at 5\% } & 0.19 & n.s & 3.57 & 0.86 & 0.71 & n.s & n.s & 0.062 & 0.116 & 0.40 & 0.02 & 0.42 \\
\hline
\end{tabular}


Table 4: Effect of interaction between nitrogen fertilizer $\mathrm{x}$ organic fertilizer on yield, its components and chemical composition of wheat (Average of 2017/2018and 2018/2019seasons).

\begin{tabular}{|c|c|c|c|c|c|c|c|c|c|c|c|c|c|}
\hline \multicolumn{2}{|c|}{$\begin{array}{l}\text { Characters } \\
\text { Treatments }\end{array}$} & $\begin{array}{c}\text { Plant } \\
\text { height } \\
\text { (cm) }\end{array}$ & $\begin{array}{c}\text { No. of } \\
\text { tillers } \\
\left(\mathbf{m}^{2}\right)\end{array}$ & $\begin{array}{c}\text { No. of } \\
\text { spikes } \\
\left(\mathbf{m}^{2}\right)\end{array}$ & $\begin{array}{c}\begin{array}{c}\text { Weight } \\
\text { of }\end{array} \\
\text { spikes } \\
\left(\mathrm{g} / \mathbf{m}^{2}\right) \\
\end{array}$ & $\begin{array}{c}\text { Gain } \\
\text { yield } \\
\left(\mathrm{g} / \mathrm{m}^{2}\right)\end{array}$ & $\begin{array}{c}\text { Grain } \\
\text { index } \\
(\mathrm{g})\end{array}$ & $\begin{array}{c}\text { Straw } \\
\text { yield } \\
(\text { ton} / \text { fed })\end{array}$ & $\begin{array}{c}\text { Grain } \\
\text { yield } \\
\text { (ton/fed) }\end{array}$ & $\begin{array}{c}\text { Biological } \\
\text { yield } \\
\text { (ton/fed) }\end{array}$ & $\begin{array}{c}\text { Harvest } \\
\text { index } \\
\%\end{array}$ & $\begin{array}{l}\text { Protein } \\
\%\end{array}$ & $\begin{array}{c}\text { Carbohydrate } \\
\%\end{array}$ \\
\hline \multicolumn{14}{|c|}{ Nitrogen fertilizer(Ammonia Gas) $x$ Organic fertilizer } \\
\hline \multirow{3}{*}{ Control } & 5 ton/ fed. & 116.75 & 519.35 & 497.47 & 759.42 & 550.02 & 42.17 & 8.515 & 4.805 & 13.320 & 36.15 & 11.40 & 78.38 \\
\hline & 10 ton/ fed. & 120.07 & 529.58 & 505.78 & 776.05 & 568.85 & 43.52 & 8.630 & 5.340 & 13.970 & 38.13 & 11.47 & 80.97 \\
\hline & 15 ton/ fed. & 123.53 & 538.10 & 518.67 & 783.90 & 581.88 & 44.95 & 8.842 & 5.267 & 14.109 & 37.30 & 11.53 & 83.75 \\
\hline \multirow{3}{*}{$\begin{array}{l}50 \mathrm{~kg} \\
\text { N/ fed. }\end{array}$} & 5 ton/ fed. & 119.97 & 530.78 & 511.87 & 774.32 & 565.30 & 43.72 & 8.713 & 5.188 & 13.901 & 37.32 & 11.47 & 80.93 \\
\hline & 10 ton/ fed. & 123.42 & 545.32 & 519.62 & 792.70 & 582.08 & 45.37 & 8.823 & 5.290 & 14.113 & 37.82 & 11.52 & 84.45 \\
\hline & 15 ton/ fed. & 126.78 & 550.28 & 528.82 & 804.87 & 594.52 & 46.97 & 8.968 & 5.377 & 14.345 & 37.52 & 11.58 & 89.17 \\
\hline \multirow{3}{*}{$\begin{array}{l}75 \mathrm{~kg} \\
\mathrm{~N} / \mathrm{fed} .\end{array}$} & 5 ton/ fed. & 123.10 & 543.87 & 527.45 & 785.37 & 576.80 & 44.63 & 8.810 & 5.228 & 14.038 & 37.30 & 11.51 & 83.50 \\
\hline & 10 ton/ fed. & 126.22 & 556.12 & 536.92 & 801.42 & 589.68 & 46.72 & 8.967 & 5.315 & 14.282 & 37.23 & 11.57 & 86.03 \\
\hline & 15 ton/ fed. & 128.77 & 568.37 & 549.13 & 812.65 & 596.07 & 48.48 & 9.070 & 5.375 & 14.445 & 37.23 & 11.70 & 88.82 \\
\hline \multicolumn{2}{|c|}{ L.S.D. at 5\% } & 0.24 & 3.24 & n.s & 1.05 & 0.87 & 0.44 & n.s & 0.076 & 0.142 & 0.49 & 0.03 & 0.51 \\
\hline
\end{tabular}


Table 5: Effect of interaction between cultivars $x$ nitrogen fertilizer $x$ organic fertilizer on yield, its components and chemical composition of wheat (Average of 2017/2018 and $2018 / 2019$ seasons).

\begin{tabular}{|c|c|c|c|c|c|c|c|c|c|c|c|c|c|c|}
\hline & \multicolumn{2}{|c|}{$\begin{array}{l}\text { Characters } \\
\text { Treatments }\end{array}$} & $\begin{array}{c}\text { Plant } \\
\text { height } \\
(\mathrm{cm})\end{array}$ & $\begin{array}{c}\begin{array}{l}\text { No. of } \\
\text { tillers } \\
\left(\mathbf{m}^{2}\right)\end{array} \\
\end{array}$ & $\begin{array}{c}\text { No. of } \\
\text { spikes } \\
\left(\mathbf{m}^{2}\right) \\
\end{array}$ & $\begin{array}{c}\text { Weight of } \\
\text { spikes } \\
\left(\mathrm{g} / \mathbf{m}^{2}\right) \\
\end{array}$ & $\begin{array}{c}\text { Gain } \\
\text { yield } \\
\left(\mathrm{g} / \mathbf{m}^{2}\right) \\
\end{array}$ & $\begin{array}{c}\text { Grain } \\
\text { index } \\
(\mathrm{g}) \\
\end{array}$ & $\begin{array}{c}\text { Straw } \\
\text { yield } \\
\text { (ton/fed) }\end{array}$ & $\begin{array}{c}\text { Grain } \\
\text { yield } \\
\text { (ton/fed) }\end{array}$ & $\begin{array}{c}\text { Biological } \\
\text { yield } \\
\text { (ton/fed) } \\
\end{array}$ & $\begin{array}{c}\text { Harvest } \\
\text { index } \\
\% \\
\end{array}$ & $\begin{array}{l}\text { Protein } \\
\%\end{array}$ & $\begin{array}{c}\text { Carbohydrate } \\
\%\end{array}$ \\
\hline \multirow{10}{*}{$\begin{array}{c}\text { Sids } \\
1\end{array}$} & \multicolumn{14}{|c|}{ Cultivars $x$ Nitrogen fertilizer(Ammonia Gas) $x$ Organic fertilizer } \\
\hline & \multirow{3}{*}{ Control } & 5 ton/ fed. & 113.40 & 516.47 & 492.43 & 751.77 & 547.70 & 41.43 & 8.470 & 4.480 & 12.950 & 34.60 & 11.38 & 76.07 \\
\hline & & 10 ton/ fed. & 116.33 & 524.83 & 495.37 & 763.47 & 558.73 & 42.43 & 8.603 & 5.437 & 14.040 & 38.30 & 11.44 & 79.13 \\
\hline & & 15 ton/ fed. & 120.93 & 533.27 & 504.07 & 774.43 & 574.03 & 43.47 & 8.797 & 5.307 & 14.104 & 37.50 & 11.51 & 82.50 \\
\hline & \multirow{3}{*}{$\begin{array}{l}50 \mathrm{~kg} \mathrm{~N} / \\
\text { fed. }\end{array}$} & 5 ton/ fed. & 116.93 & 527.63 & 510.40 & 765.20 & 562.00 & 42.47 & 8.650 & 5.193 & 13.843 & 37.50 & 11.45 & 77.80 \\
\hline & & 10 ton/ fed. & 120.10 & 543.83 & 517.57 & 784.60 & 573.67 & 44.47 & 8.743 & 5.323 & 14.066 & 38.40 & 11.48 & 83.40 \\
\hline & & 15 ton/ fed. & 124.83 & 553.37 & 523.00 & 798.17 & 592.00 & 46.53 & 8.827 & 5.383 & 14.210 & 37.90 & 11.53 & 88.83 \\
\hline & \multirow{3}{*}{$\begin{array}{l}75 \mathrm{~kg} \mathrm{~N} / \\
\text { fed. }\end{array}$} & 5 ton/ fed. & 120.20 & 543.67 & 528.53 & 773.13 & 577.10 & 43.53 & 8.680 & 5.180 & 13.860 & 37.47 & 11.51 & 81.50 \\
\hline & & 10 ton/ fed. & 123.93 & 555.17 & 534.10 & 788.27 & 590.40 & 45.53 & 8.860 & 5.243 & 14.103 & 37.20 & 11.51 & 84.60 \\
\hline & & 15 ton/ fed. & 126.57 & 568.10 & 546.70 & 804.93 & 596.43 & 47.63 & 8.950 & 5.327 & 14.277 & 37.33 & 11.68 & 87.73 \\
\hline \multirow{10}{*}{$\begin{array}{c}\text { Misr } \\
1\end{array}$} & \multirow{3}{*}{ Control } & 5 ton/ fed. & 120.10 & 522.23 & 502.50 & 767.07 & 552.33 & 42.90 & 8.560 & 5.130 & 13.690 & 37.70 & 11.42 & 80.70 \\
\hline & & 10 ton/ fed. & 123.80 & 534.33 & 516.20 & 788.63 & 578.97 & 44.60 & 8.657 & 5.243 & 13.810 & 37.97 & 11.49 & 82.80 \\
\hline & & 15 ton/ fed. & 126.13 & 542.93 & 533.27 & 793.37 & 589.73 & 46.43 & 8.887 & 5.227 & 14.114 & 37.10 & 11.54 & 85.00 \\
\hline & \multirow{3}{*}{$\begin{array}{l}50 \mathrm{~kg} \mathrm{~N} / \\
\text { fed. }\end{array}$} & 5 ton/ fed. & 123.00 & 533.93 & 513.33 & 783.43 & 568.60 & 44.97 & 8.777 & 5.183 & 13.960 & 37.13 & 11.49 & 84.07 \\
\hline & & 10 ton/ fed. & 126.73 & 546.80 & 521.67 & 800.80 & 590.50 & 46.27 & 8.903 & 5.257 & 14.160 & 37.23 & 11.56 & 85.50 \\
\hline & & 15 ton/ fed. & 128.73 & 547.20 & 534.63 & 811.57 & 597.03 & 47.40 & 9.110 & 5.370 & 14.480 & 37.13 & 11.62 & 89.50 \\
\hline & \multirow{3}{*}{$\begin{array}{l}75 \mathrm{~kg} \mathrm{~N} / \\
\text { fed. }\end{array}$} & 5 ton $/ \mathrm{fed}$. & 126.00 & 544.07 & 526.37 & 797.60 & 576.50 & 45.73 & 8.940 & 5.277 & 14.217 & 37.13 & 11.52 & 85.50 \\
\hline & & 10 ton/ fed. & 128.50 & 557.07 & 539.73 & 814.57 & 588.97 & 47.90 & 9.073 & 5.387 & 14.460 & 37.27 & 11.63 & 87.47 \\
\hline & & 15 ton/ fed. & 130.97 & 568.63 & 551.57 & 820.37 & 595.70 & 49.33 & 9.190 & 5.423 & 14.613 & 37.13 & 11.72 & 89.90 \\
\hline & \multicolumn{2}{|c|}{ L.S.D. at 5\% } & 0.34 & 4.58 & n.s & 1.49 & 1.24 & 0.62 & n.s & 0.107 & 0.201 & 0.69 & n.s & 0.73 \\
\hline
\end{tabular}


grain yield (ton/fed.), biological yield (ton/fed.) and harvest index was significant in both seasons, while was not significant in grain index (g), straw yield ton/feddan, protein percentage and carbohydrate percentage. Data in Table (2) indicated that the highest value of all characters under study was Miser-1 cultivar $+75 \mathrm{~kg} \mathrm{~N} /$ feddan, except grain yield $\left(\mathrm{g} / \mathrm{m}^{2}\right)$ and harvest index in both seasons. Results were in accordance with those obtained by Zaki et al. (2012).

Data in Table (3) illustrated that the most effective treatment for plant height $(\mathrm{cm})$, number of spikes $/ \mathrm{m}^{2}$, weight of spikes $\left(\mathrm{g} / \mathrm{m}^{2}\right)$, grain yield $\left(\mathrm{g} / \mathrm{m}^{2}\right)$, grain yield (ton/fed.), biological yield (ton/fed.) protein percentage and carbohydrate percentage was Miser-1 cultivar with 15 ton / feddan organic fertilizer. In the same time Sids-1 cultiver +10 ton / feddan organic fertilizer gave the highest value of harvest index.

Table (4) revealed that treatment of $75 \mathrm{~kg} \mathrm{~N} /$ feddan +15 ton / feddan organic fertilizer gave the highest value of all characters under study in both seasons except harvest index.

Data in Table (5) show clearly that yield and yield components of wheat plants were significantly affected by interaction between wheat cultivars $\mathrm{x}$ nitrogen fertilizer $\mathrm{x}$ organic fertilizer in both seasons except number of spikes/ $\mathrm{m}^{2}$, straw yield ton / feddan and protein percentage. Tallest plants, the highest number of tillers $\left(\mathrm{m}^{2}\right)$, weight of spikes $\left(\mathrm{g} / \mathrm{m}^{2}\right)$, grain index $(\mathrm{g})$, grain yield (ton/fed.), biological yield (ton/fed.) and carbohydrate percentage were obtain by using Misr- 1 cultivar $+75 \mathrm{~kg} \mathrm{~N}$ / fed., +15 ton / fed., organic fertilizer. On the other hand the highest grain yield $\left(\mathrm{g} / \mathrm{m}^{2}\right)$ was obtained by using Misr-1 cultivar $+50 \mathrm{~kg} \mathrm{~N} /$ fed., +15 ton / fed., organic fertilizer.

\section{References}

Abd El-All, A. E. A.; E. Ghalab and Asal M. Wali, 2017. Effect of Nitrogen and Potassium Fertilization on Yield and Nitrogen Use Efficiency of Two Wheat Cultivars Grown under Sprinkler Irrigation in Sandy Soil. J. Soil Sci. and Agric. Eng., Mansoura Univ., 8 (12): 693 - 702.

Abd El-Gawad, A.A., K.A. El-Shouny, S.A. Saleh and M.A. Ahmed, 1987. Partition and migration of dry matter in newly cultivated wheat varieties. Egypt. J. Agron., 12(1-2): 1-16.

Ahmed, M.A., K.A. Aboud and M.S. Hassanein, 2013. Genetic Analysis of Energy Production in Nine Wheat Cultivars Cultivated in Egypt. J. of Appl. Sci. Res., 9(8): 4558-4566.

A.O.A.C., 1988. Official Methods of Analysis of the Association of official Analytical Chemists 21th Ed-Washington D.C.

Chapman, H.D. and P.F. Pratt, 1961. Methods of Analysis for Soils, Plants and Water. Univ. of California, Berkeley, CA, USA.

Dubois, M., K.A. Gilles. J.K. Hamilton, P.A. Rebers and F. Smith, 1956. Colorimetric method for determination of sugars and related substances. Anal. Chem., 28: 350-356.

El-Esh, I.F.I., 2007. Effect of both mineral and biofertilization on yield and quality of Wheat. Ph. D. Thesis, Fac. Agric. Alex., Univ., Egypt.

Gomez, A.K. and A.A. Gomez, 1984. Statistical procedure for agricultural Research.2nd edition John Wiley and Sons, Inc., New York.

Hassanein, M. S., Amal G. Ahmed and Nabila M. Zaki, 2018. Effect of nitrogen fertilizer and biofertilizer on yield and yield components of two wheat cultivars under sandy soil. Middle East J. of Appli. Sci., 8(1):37-42.

Hassanein, M.S., Amal G. Ahmed, Nabila M. Zaki, H.I. El-Aila and M.M. Tawfic, 2013. Response of two wheat cultivars grown in newly cultivated lands to iron and slow release $\mathrm{N}$ fertilizers. Aust. J. of Basic and Appl. Sci., 7(1): 498-505.

Hassanein M. S., Nabila M. Zaki and Amal G. Ahmed, 2019. Effect of foliar nutrition micro mix fertilizer on yield and yield components of two wheat cultivars. Middle East J. Appl. Sci., 9(1): 86-90.

Hosam El-Din, A.T.S., 2007. Productivity of some wheat varieties by using bio and organic fertilization in the New Valley. M. Sc. Thesis, Fac. Of Agric. Ain Shams Univ., Egypt.

Kabesh, M.O., M.F. El-Kramany, G.A. Sary, H.M. El-Naggar and Gehan, Sh. H. Bakhoum, 2009. Effect of sowing Methods and some bio-organic fertilization treatments on yield and yield components of wheat. Res. J. Agric. \& Biol. Sci., 5(1): 97-102. 
Sabah, H. Abo-El-Ela, 2006. Influence of mineral and bio-nitrogen fertilization on three new bread wheat genotypes. Egypt. J. Agric. Res., 84(6): 1833-1841.

Sadur, Rehman; S.K. Khalil, Abdur Rehman and A.U.R. Saljoqi, 2008. Organic and inorganic fertilizers increase wheat yield components and biomass under rainfed condition. Sarhad J. Agric., 24(1): 11- 20.

Shoman, H.A., A.M. Abo-Shtaia, K.A. El-Shouny and M.A. Abd El-Gawad, 2006. Effect of biological and organic fertilization on yield and its components of two wheat cultivars under Al-Wadi AlGadeed conditions. Alex. J. Agric. Res., 51: 49-65.

Suzan, A.K.I., 2007. Effect of auxin levels and phosphorus biofertilization on wheat yield and its components. M. Sc. Thesis, Fac. of Agric. Alexandria Univ., Egypt.

Wali A. M., Abdelaal Shamseldin, F. I. Radwan, E. M. Abd El Lateef and N. M. Zaki, 2018. Response of Barley (Hordeum vulgare) Cultivars to Humic Acid, Mineral and Biofertilization under Calcareous Soil Conditions. Middle East Journal of Agriculture, 7 (1):71-82.

Zaki, N. M., Amal G. Ahmed and M. S. Hassanein, 2019. Effect of cultivars, nitrogen fertilizer and biofertilizer on yield and yield components on barley. Current Sci. Int., 8(2): 299-306.

Zaki, N.M., Amal G. Ahmed, M.S. Hassanein and Mirvat E. Gobarah, 2015. Response of two wheat cultivars to foliar fertilizer in newly cultivated land. Middle East J. of Agric. Res., 4 (2): 283290.

Zaki, N.M., M.A. Gomaa, F.I. Radwan, M.S. Hassanein and A.M. Wali, 2012. Effect of mineral, organic and bio-fertilizers on yield, yield components and chemical composition of some wheat cultivars. J. of Applied Sci. Res., 8 (1): 174-191.

Zaki N. M., M. S. Hassanein and Amal G. Ahmed, 2018. Effect of foliar nutrition grow vit on yield and yield components of two wheat cultivars. Middle East J. of Agric. Res., 7 (2): 248- 253.

Zaki, N. M., M.S. Hassanein, Amal G. Ahmed, M.A. Ahmed and M.M. Tawifk, 2016. Response of two wheat cultivars to different nitrogen sources in newly cultivated land. Res. J. of Pharmaceutical, Biolog. and Chem. Sci., 7 (6): 410-416.

Zeidan, E.M., I.M. Abd El-Hameed, A.H. Bassiouny and A.A. Waly, 2009. Effect of irrigation intervals, nitrogen and organic fertilization on yield, yield attributes and crude protein content of some wheat cultivars under newly reclaimed saline soil conditions. 4th Conference on Recent Technologies in Agriculture, 2009. 